\title{
Aspectos bioecológicos y de manejo de gallina ciega (Phyllophaga sp.), en la producción de cacao (Theobroma cacao) en Río San Juan, Nicaragua
}

\section{Bioecology and management of white grub (Phyllophaga sp.), in the production of cocoa (Theobroma cacao), Río San Juan, Nicaragua}

\section{Harold Argüello-Chávez}

Docente Investigador, Departamento de Protección Agrícola y Forestal, Facultad de Agronomía, Universidad Nacional Agraria. harold.arguello@ci.una.edu.ni

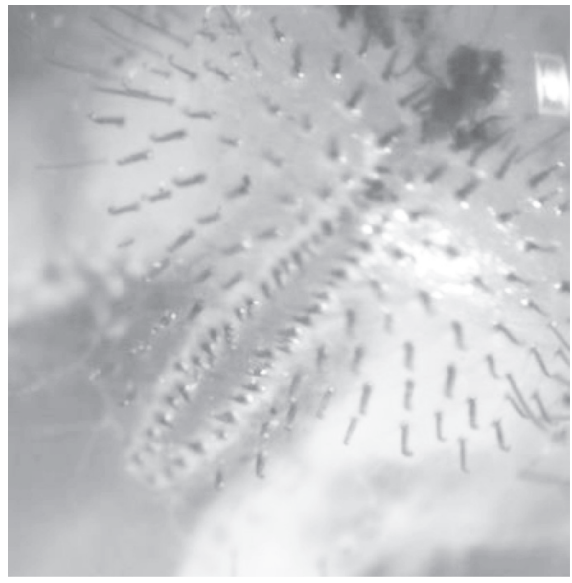

\section{RESUMEN}

La gallina ciega (Phyllophaga sp.) es la plaga de suelo predominante en cultivos de Centroamérica, afecta al cacao (Theobroma cacao), y a gran cantidad de cultivos de diferentes familias botánicas. No existe registro de variedades de cacao tolerante al ataque de gallina ciega y las alternativas de manejo se centran en la aplicación de químicos sintéticos, cuyos efectos residuales en suelos y cultivos no son compatibles con los requerimientos fitosanitarios establecidos por las agencias certificadoras. El objetivo del presente trabajo es documentar la problemática ocasionada por gallina ciega en plantaciones de Cacao en la Región Autónoma del Atlántico Sur (RAAS), basado en estudio de campo realizado para el reconocimiento de especies, incidencia, severidad, caracterización de daños, distribución poblacional y diseño de una estrategia de manejo de gallina ciega en la finca San Sebastián, ubicada al noroeste de la comunidad Buena Vista, del municipio Baca de Sábalo en el departamento de Río San Juan en diciembre del 2016. Se enfatiza sobre algunos aspectos taxonómicos y biológicos de la plaga que la hace predecible para enfocar un manejo adecuado de la misma; se proponen recomendaciones acerca de estrategias de manejo sostenible de la plaga y se enfatiza sobre premisas o aspectos técnicos básicos a tomar en cuenta para diseñar una eficiente estrategia de manejo de gallina ciega a mediano y largo plazo.

Palabras clave: gallina ciega, daño, cacao, manejo.

\section{ABSTRACT}

White grubs (Phyllophaga sp.) is the predominant soil pest in Central American crops, it affects cacao (Theobroma cacao), and a large number of crops from different botanical families. There is no record of varieties of cacao tolerant to white grubs attack and the management alternatives are focused on the application of synthetic chemicals, whose residual effects on soils and crops are not compatible with the phytosanitary requirements established by the certifying agencies. The objective of this paper is to document the problems caused by white grubs in cocoa plantations in the South Atlantic Autonomous Region (RAAS), based on a field study carried out for the recognition of species, incidence, severity, damage characterization, population distribution and design of a white grubs management strategy in the San Sebastian farm, located northwest of the Buena Vista community, in the municipality of Boca de Sabalo in the department of Río San Juan in December 2016. Some taxonomic and biological aspects are emphasized of the plague that makes it predictable to focus on an adequate management of it; recommendations about strategies for sustainable management of the pest are proposed and emphasis is placed on premises or basic technical aspects to be taken into account in order to design an efficient white grubs management strategy for the medium and long term.

Keywords: White grubs, damage, cocoa, management.
Recibido: 20 de junio 2017

Aceptado: 6 de noviembre del 2017
Los artículos de la revista La Calera de la Universidad Nacional Agraria, Nicaragua, se comparten bajo términos de la licencia Creative Commons: Reconocimiento, No Comercial, Compartir Igual. Las autorizaciones adicionales a las aquí delimitadas se pueden obtener en el correo freddy.aleman@ci.una.edu.ni Universidad Nacional Agraria 


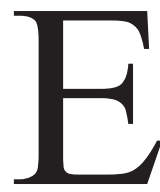
1 Cacao fue un producto de consumo privilegiado en las estructuras sociales, religiosas, políticas y militares antes de la colonización española, al punto de ser un símbolo de valor comercial intercambiable.

A pesar de que aporta menos del uno por ciento a las exportaciones en la economía mundial (CATIE, 2009), para la región centroamericana representa un rubro importante para la diversificación productiva. Datos macroeconómicos conceden al cacao un incremento constante para mercados internacionales estimulados por cifras que indican que el área de cacao sembrada en Centroamérica ha aumentado un $22 \%$ en los últimos 10 años (CAMBio, 2015).

Lo anterior, demuestra el interés creciente en Centroamérica y de países como Nicaragua por producir y comercializar cacao con características diferenciales para el mercado internacional. En Nicaragua el área sembrada asciende a 14 mil 600 hectáreas (CAMBio, 2015), lo que representa un incremento del 12 por ciento en la última década. El aumento ha sido sobre todo en zonas de la Región Autónoma del Atlántico Sur (RAAS) y el centro norte del país.

Entre 2000 y 2010 el incremento en el volumen exportable de cacao ha sido más de 1000 por ciento (CETREX, 2010). Las condiciones previas de uso de suelo en las áreas cacaoteras han sido ganaderas y forestales; lo que las hace susceptibles a ser afectadas por poblaciones de plagas de suelo, principalmente Phyllophaga sp., mejor conocida como gallina ciega, muy propia de estas zonas productivas.

La gallina ciega es la plaga de suelo predominante en cultivos de Centroamérica, afecta el cacao, y gran cantidad de cultivos de diferentes familias botánicas. Los daños causados varían entre 10 y hasta 100 por ciento de las plantaciones (King, 1984; King y Saunders, 1984, Argüello, Cáceres y Monzón, 1997). El daño principal lo causa al alimentarse activamente de las raíces. Además provoca daños secundarios al alimentarse parcialmente y provoca lesiones por su daño, las cuales son una vía de introducción de patógenos del suelo, como bacterias y hongos. La gallina ciega se encuentra en casi todos los hábitats donde se establecen cultivos, desde 50 hasta 1750 metros sobre el nivel del mar.

Los adultos vuelan sobre áreas relativamente pequeñas, no son insectos invasores, por lo que generaciones de ellos ocupan un micro clima en el que se reproducen constantemente. Las condiciones de alta humedad en casi todo el año en las zonas de la Región Autónoma del Atlántico Sur (RAAS), favorecen la permanencia de vegetación que ofrece el alimento que estos insectos necesitan para colonizar exitosamente las áreas de cultivos o zonas aledañas.

La gallina ciega es un insecto con ciclo de vida complejo divido en cuatro etapas: huevo, larva (gusano o joboto), pupa (cartucho) y adulto (ronrón). La larva se desarrolla por medio de tres instares, en el tercero y último es que se alimenta activamente de raíces durante 4 o 5 meses, tiempo en el cual la larva provoca el mayor daño a los cultivos. La época en la que se presentan los daños a las raíces en las plantaciones de cacao, está relacionado al tipo de especie de gallina ciega predominante en la zona de cultivo.

El objetivo del presente trabajo es documentar la problemática ocasionada por gallina ciega en plantaciones de Cacao en la Región Autónoma del Atlántico Sur (RAAS), basado en estudio de campo realizado para el reconocimiento de especies, incidencia, severidad, caracterización de daños, distribución poblacional y diseño de una estrategia de manejo de gallina ciega en la finca San Sebastián, ubicada al noroeste de la comunidad Buena Vista, del municipio Baca de Sábalo en el departamento de Río San Juan en diciembre del 2016; se enfatiza sobre algunos aspectos taxonómicos y biológicos de la gallina ciega que la hace predecible para enfocar un manejo adecuado de la misma; se dan recomendaciones acerca de estrategias de manejo sostenible de la plaga y se recalca acerca de premisas o aspectos técnicos básicos a tomar en cuenta para diseñar una eficiente estrategia de manejo de gallina ciega a mediano y largo plazo.

\section{MATERIALES Y MÉTODOS}

Agroindustrial Del Río S.A es una empresa productora y procesadora principalmente de Cacao, administra la finca "San Sebastián", está ubicada en dirección noroeste de la comunidad Buena Vista del municipio de Sábalo, en el departamento Rio San Juan. La finca consta de dos unidades productivas de cacao y las plantaciones provienen de diferentes orígenes y diverso germoplasma; tienen 3 años de sembradas, existen lotes en evaluación que no han sido sembrados todavía. El tipo de manejo agronómico y fitosanitario que se le aplica a las plantaciones es Convencional.

Se seleccionó la zona con lotes ya sembrados, la cual consiste de 12 lotes con diferentes dimensiones. Se priorizó el registro de lotes con mayor incidencia de gallinas ciegas, por ello se muestrearon los lotes 11 con 5.37 ha; lote 10 con 6 ha; lote 9 con 4.27 ha, lote 6 con 5 ha y lote 2 con 4.7 ha; en total se registraron 25.35 ha en 5 lotes de 12 que constituyen la parte inicial de la finca.

El muestreo del pie cuadrado fue escogido para evaluar la población de larvas de gallina ciega presentes. De los lotes con mayor tamaño $(11,10$ y 9) se seleccionaron 10 puntos (en posturas de cultivos y en calle); los lotes con menor dimensión ( 6 y 2) se escogieron 8 puntos todos al azar y con distribución representativa.

Se tomó cada espécimen encontrado en cada hoyo colocándose en una bolsa rotulada y con tierra para sobrevivencia de las larvas; se evitó tomar las larvas directamente con la mano dado que esto las maltrata y al mantenerse en bolsa con tierra no duran más de dos días. Se tomaron muestras de plantas afectadas a diferentes niveles de severidad por gallina ciega.

Se reconocieron los síntomas y daños que causa la alimentación de gallina ciega en las raíces de plantas de cacao establecidos en diferentes fechas de siembra. La severidad de los síntomas está relacionada directamente a la cantidad de raíces afectadas por la plaga. La distribución de la bárbula y el arreglo de los dientecillos del ráster son las características morfológicas que se toman para realizar el diagnóstico preliminar de las especies de gallina ciega, estas estructuras están ubicadas en la zona dorsal del ano de cada larva. Las características no son consistentes entre las mismas especies, es decir podrá existir una mínima variante morfológica entre uno o varios especímenes de una misma especie, por ello se determina identificación preliminar. Se compara la lista de 
especies reconocidas por medio de las larvas con los adultos que serán capturados e identificados al momento que salen en vuelo en las primeras semanas de mayo o al inicio de las primeras lluvias del próximo año, y con ello finalmente confirmar el diagnóstico de especies de gallina ciega presentes.

El diseño de una estrategia de manejo sostenible de gallina ciega, requiere reconocer los síntomas y daños que causa gallina ciega en las raíces de plantas de cacao durante los primeros meses posterior al trasplante. Estos daños son provocados indistintamente de la edad de la plantación. La severidad de los síntomas está relacionada directamente a la cantidad de raíces afectadas por la plaga (Argüello y Lastres, 2012). Los síntomas iniciales se expresan en cambio de coloración en el follaje.

La estrategia de manejo sostenible sugerida requiere categorización de lotes por niveles poblacionales de larvas presentes en el último muestreo realizado. Esta categorización es a base de colores (semáforo) los cuales indican el nivel severidad del daño presente en cada lote o plantación. Los colores indicativos son rojo (nivel más alto), amarillo (nivel intermedio) y verde (nivel más bajo).

Para analizar la severidad del daño, se valoran cuatro criterios simultáneos (condición de tallo, foliar, radicular y nivel poblacional) en tres categorías de severidad diferentes (daño inicial, daño intermedio y daño irreversible).

Para cada lote categorizado, se identifican prácticas y labores a implementar con el propósito disminuir progresivamente promedios poblacionales y por consiguiente los porcentajes de daños registrados en el corto, medio y largo plazo.

\section{RESULTADOS Y DISCUSIÓN}

La revisión de larvas del tercer instar registran la presencia de dos subfamilias (Coleóptera: Scarabeidae): Melolonthinae representado por el género Phyllophaga y Rutelinae representada por los géneros Anomala y Cyclocephala. Las especies de gallina ciega predominantes en las zonas de la RAAS y Centro Norte de Nicaragua son: Phyllophaga elenans, bianual (SAYLOR, 1938), P. valeriana, anual (SAYLOR, 1934), P. vicina, anual y bianual), (MOSER 1918), y P. obsoleta, anual (BLANCHARD, 1850).

Existen identificadas más de 20 especies de gallina ciega en Nicaragua (Argue1lo, Caceras y Morón, 1998; Maes y Morón, 2017), las cuales presentan dos tipos de ciclos, anual y bianual. Las especies bianuales se caracterizan por provocar daños en las plantaciones en la época de siembra de primera, entre los meses de mayo y agosto. Las especies anuales lo hacen en la época de postrera, entre septiembre y diciembre. La convergencia poblacional de especies con ambos comportamientos puede generar daños en períodos de tiempo prolongados, es decir tanto en primera como en postrera.

La identificación taxonómica preliminar de las especies de gallina ciega es estado larvario está basado en la distribución de la bárbula y el arreglo de los dientecillos del ráster. Estas son las características morfológicas que se toman en cuenta para realizar el diagnóstico preliminar. Las estructuras están ubicadas en la zona dorsal del ano de cada larva. Las características no son consistentes entre las mismas especies, es decir podrá existir una mínima variante morfológica entre uno o varios especímenes de una misma especie, por ello se determina identificación preliminar (Argüello, Cáceres y Morón, 1998).

La figura 1, resalta las especies de gallina ciega asociadas a plantaciones de Cacao establecidas en la zona Región Autónoma del Atlántico Sur (RAAS), y detalles de cada una de las especies.
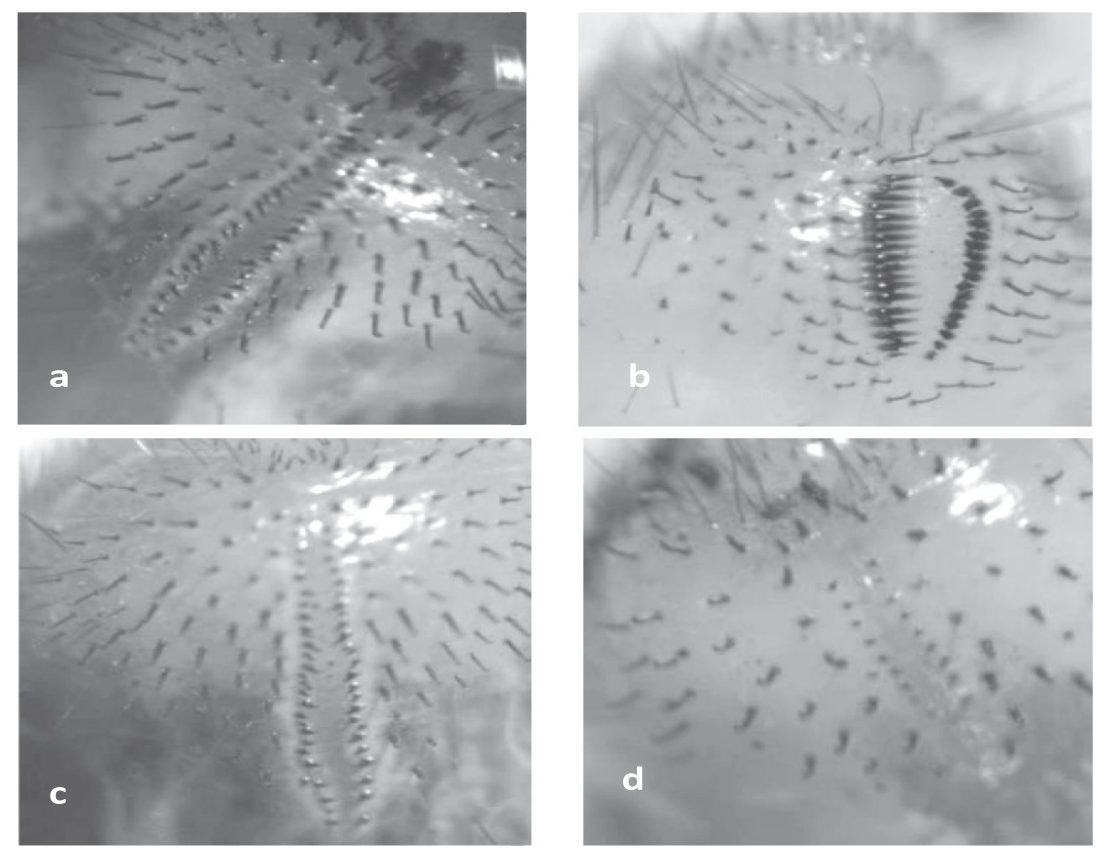

Figura 1. Especies de gallina ciega asociadas a plantaciones de Cacao establecidas en la zona Región Autónoma del Atlántico Sur (RAAS). Detalles del ráster en larvas proyectadas dorso ventralmente. (1a). Phyllophaga elenans (SAYLOR, 1938). Es la especie predominante, además de encontrarse en todas las zonas, supera en número a las otras especies. Posee comportamiento bianual, con larvas L3 de tamaño relativamente superior, su capacidad de alimentación más agresiva hace que sea predominante en las zonas de presencia superando en 6 a 1 comparativamente a las otras especies; cohabita lugares menores a los $300 \mathrm{msnm}$. Generalmente se evidencia altas poblaciones y mayor daño en años alternos. (1b). P. valeriana (SAYLOR, 1934. Especie de registros en zonas mayores a los $1000 \mathrm{msnm}$, de comportamiento anual, no se ha reportado como especie principal en lugares con mayor incidencia de la plaga. En este caso se encontró un solo espécimen. (1c). P. vicina (MOSER 1918). Especie registrada con hábito facultativo, es decir puede comportarse como anual o bianual, se colectaron dos especímenes lo cual indica su poca importancia en provocar daño a las plantas. (1d). P. obsoleta. (BLANCHARD, 1850). Especie reportada como anual, regularmente prefiere cohabitar sitios mayores a los 1000, no obstante, se ha reportado en lugares más bajos. No representa riesgo de daño por presentar bajas poblaciones. 
El monitoreo y descripción de los síntomas iniciales por daño de gallina ciega en raíces de cacao indican que se expresan en el cambio de coloración foliar, las hojas se tornan de color verde oscuro a verde pálido y de verde pálido en amarillo. La coloración amarilla indica que el sistema radicular fue eliminado en más del $50 \%$, en algunos casos se registra directamente daño a la raíz pivotante y ausencia de pelos absorbentes. El comportamiento foliar se muestra progresivo, en la medida que el daño aumenta la cantidad de hojas disminuye, aunque el grosor del tallo y el tamaño de la planta se mantiene.

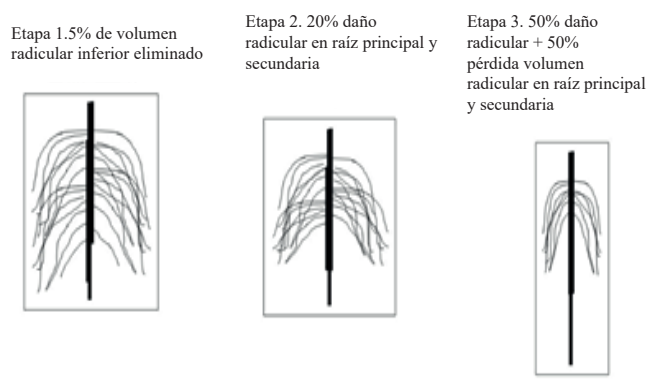

Figura 2. Escala grafica de daño provocado por gallina ciega en raíces de plantas de cacao.

El sistema de muestreo en campo para áreas extensivas requiere zonificar las áreas productivas para registrar niveles poblacionales y con ello definir estrategias específicas, debido a que la concentración poblacional no es uniforme en todos los lotes o sectores de las plantaciones de cacao. El muestreo debe iniciar antes del establecimiento de las plantas de cacao. El tipo de muestreo a desarrollar para evaluar las poblaciones de gallina ciega en campo es el de pie cuadrado. Este tipo de muestreo permite estimar la concentración de gusanos por área y la distribución poblacional en la zona afectada (PROMIPAC, 2006).

Premisas o aspectos técnicos básicos a tomar en cuenta para diseñar una eficiente estrategia de manejo de gallina ciega a mediano y largo plazo. Los insecticidas depositados en suelos con saturación de humedad ofrecen reducida capacidad de protección, dado que no logran penetrar eficientemente la rizósfera; debido a los pocos espacios para la libertad de movimiento vertical de los ingredientes activos en el suelo.

Por otro lado, la efectividad de un tratamiento se determina por la progresión o reducción de la incidencia del insecto en plantas, es decir el tratamiento que consiga mejor efecto reduce el nivel inicial de incidencia en el tiempo de la plaga, con lo cual afecta nuevas plantas o nuevos surcos.

La población de gallina ciega presente en el suelo de un lote no se alimenta exclusivamente de raíces de cacao. El Cacao
Cuadro 1. Caracterización del daño, severidad y nivel poblacional de gallina ciega en

\begin{tabular}{lllll}
\hline Nivel de daño & Condición de tallo & Condición foliar & Condición radicular & Nivel poblacional* \\
\hline $\begin{array}{l}\text { Daño inicial } \\
\text { o mínimo (etapa 1) }\end{array}$ & $\begin{array}{l}\text { Crecimiento y } \\
\text { grosor normal }\end{array}$ & $\begin{array}{l}\text { coloración variuada } \\
\text { verde y amarilla sin } \\
\text { defoliación }\end{array}$ & $\begin{array}{l}5 \% \text { de volumen } \\
\text { radicular inferior } \\
\text { eliminado }\end{array}$ & $\leq 0.1$ \\
$\begin{array}{llll}\text { Daño intermedio } \\
\text { (etapa 2) }\end{array}$ & $\begin{array}{l}\text { Crecimiento y } \\
\text { grosor deficiente }\end{array}$ & $\begin{array}{l}\text { Coloración amarilla y } \\
\text { 20\% de perdida } \\
\text { foliar }\end{array}$ & $\begin{array}{l}20 \% \text { daño radicular } \\
\text { lesiones en raíz principal } \\
\text { y secundarias }\end{array}$ & $0.2 \geq \leq 0.4$ \\
$\begin{array}{l}\text { Daño irreversible } \\
\text { (etapa 3) }\end{array}$ & $\begin{array}{l}\text { Crecimiento y } \\
\text { grosor deficiente }\end{array}$ & $\begin{array}{l}\text { Coloración amarilla } \\
\text { severa y 50\% perdida } \\
\text { foliar }\end{array}$ & $\begin{array}{l}50 \% \text { daño radicular } \\
+50 \% \text { pérdida de volumen } \\
\text { radicular, lesiones en } \\
\text { raíz principal y secundaria. }\end{array}$ &
\end{tabular}

Cuadro 2. Estrategia de manejo de gallina ciega de acuerdo al rango poblacional (categorización de color)

\begin{tabular}{|c|c|c|}
\hline Categoría del lote & Promedio & Estrategia de manejo de gallina ciega \\
\hline Verde & $\leq 0.1$ & $\begin{array}{l}\text { Muestreo de larvas y severidad de daño cada tres meses } \\
\text { Mantenimiento de limpieza o carrileo (eliminación de plantas voluntarias de pasto, y } \\
\text { demás plantas tanto en el área de las plantas de cacao como en la calle de cada surco) } \\
\text { Aplicación de solución arrancadora (18-46-0 diluido) al momento de la siembra } \\
\text { Aplicación de cepa mezclada de Metarhiziumy Beauveria dirigida a la raíz (cada } \\
\text { tres meses) } \\
\text { Aplicación de Proroot estimulante de raíces secundarias (cada tres meses durante los } \\
\text { dos primeros años) } \\
\text { Inoculación de Micorrizas (dos veces al año) } \\
\text { Aplicación de Trichodermay Bacillus subtillis dirigida a la raiz (2 veces al año) }\end{array}$ \\
\hline Amarillo & $0.2>\leq 0.4$ & $\begin{array}{l}\text { Muestreo de larvas y severidad de daño (tres veces al año) } \\
\text { Mantenimiento de limpieza o carrileo } \\
\text { Aplicación de Proroot estimulante de raíces secundarias (cada } 6 \text { meses) } \\
\text { Aplicación de Trichodermay Bacillus subtillis dirigida a la raíz (una vez al año) } \\
\text { Aplicación de cepa mezclada de Metarhiziumy Beauveria dirigida a la raíz (cada } \\
\text { tres meses) } \\
\text { Aplicación de granulado de contacto de alta solubilidad y poca residualidad, dirigido } \\
\text { al pie de la planta. }\end{array}$ \\
\hline Rojo & $>0.8$ & $\begin{array}{l}\text { Muestreo de larvas y severidad de daño (cada tres meses) } \\
\text { Mantenimiento de limpieza o carrileo } \\
\text { Aplicación de Trichodermay Bacillus subtillis dirigida a la raíz (una vez al año) } \\
\text { Aplicación de granulado de contacto de alta solubilidad y poca residualidad, dirigida } \\
\text { a la calle y al pie de la planta } \\
\text { Aplicación de contacto-ingestión para rotación de ingrediente activo y evitar } \\
\text { resistencia en el largo plazo. } \\
\text { Resiembra de plantas afectadas }\end{array}$ \\
\hline
\end{tabular}

rango de hospederos es amplio, lo cual indica que se mantienen alimentándose de raíces de arvenses ubicadas en la calle o espacios entre surcos.

Las larvas L3, aunque presentan poca movilidad, existe movimiento de traslado del centro del surco hacia las raíces de las plantas desprotegidas, lo cual indica que de esta manera se da la reinfestación de larvas.

Los insecticidas sistémicos de absorción radicular protegen a las plantas de daño solo si los larvas se alimentan de ellas, lo cual indica que ofrecen una protección local y no general.

Insecticidas con ingredientes activos biológicos como Metarhizium sp. y Beauveria sp. para el control de gallina ciega funcionan de contacto, y su efectividad depende de varios factores fundamentales: especificidad de las cepas aplicadas; de la capacidad de colonización de las zonas de protección; de la cantidad de inóculo presente y de la humedad predominan del suelo (Altamirano, Zamora y Argüello, 2003). 
Las especies de gallina ciega de ciclo biológico bianual suelen causar daños en raíces con larvas L3 en época de primera y se afectan a las plantas en años intermedios; las especies de ciclo anual provocan daño en época de postrera.

\section{CONCLUSIONES}

En la finca San Sebastián, existe diversidad intraespecífica predominante hacia especies de gallina ciega de ciclo bianual, aunque se determina presencia de especies anual.

La prevalencia y severidad del daño en raíces cacao, indica la coexistencia del complejo de especies en el microclima Cacao.
Las prácticas y estrategias de manejo de poblaciones de gallina ciega a desarrollar en la finca San Sebastián, deberán considerar reducir poblaciones en el mediano y largo plazo.

\section{AGRADECIMIENTOS}

A la empresa Agroindustrial Del Rio, por sus aportes informativos sobre la incidencia y los daños provocados por esta plaga en plantaciones comerciales de cacao establecidos en el Departamento de Rio San Juan, y al departamento de comunicaciones de la Universidad Nacional Agraria por sus aportes en las corrección del texto.

\section{REFERENCIAS BIBLIOGRÁFICAS}

Argüello, H.; Lastres, L. (Eds). 2012. Experimentación campesina en Manejo Integrado de Plagas. Guía metodológica para facilitar procesos de experimentación campesina en Manejo Integrado de Plagas en Escuela de Campo. Programa de Manejo Integrado de Plagas en América Central. Zamorano Academic Press. Universidad Zamorano, Honduras. 2012. 105P.

Altamirano, M.; Zamora, M.; Argüello, H. 2003. Evaluación de opciones no químicas para el manejo de gallina ciega (Phyllophaga spp.) en el cultivo del repollo (Brassica oleracea). en Miraflor, Estelí. Tesis de Ingeniero Agrónomo. Universidad Nacional Agraria. Managua, Nicaragua. Pág. 94.

Argüello, H.; Cáceres, O.; Morón, M.A. 1999. Guía Ilustrada para identificación de especies de gallina ciega (Phyllophaga spp) presentes en las principales zonas agrícolas de Nicaragua. PROMIPAC-Nicaragua. Estelí, Nicaragua. p. 30.

Argüello, H.; Cáceres, O.; Monzón, A. 1998. Especies de gallina ciega (Phyllophaga spp) y sus relaciones con factores agro ecológicos en la zona norcentral de Nicaragua. Boletín Nicaragüense de Entomología. León, Nicaragua. 16 p.

Argüello, H.; Cáceres, O.; Monzón, A. 1999. Validación de trampas lumínicas artesanales para el control de adultos de gallina ciega (Phyllophaga spp) en Almaciguera y Miraflor, Estelí, Nicaragua. Boletín Nicaragüense de Entomología. León, Nicaragua. 9 p.

Argüello, H.; Caceres, O. 1998. Taxonomía de especies de gallina ciega (Phyllophaga spp) presentes en la parte norcentral de Nicaragua. PROMIPAC Nicaragua y Universidad nacional Agraria. Estelí, Nicaragua. 13 p.

Argüello, H.; Cáceres, O.; Monzon, A. 1997. Inventario Agro ecológico de especies de gallina ciega (Phyllophaga spp) presentes en la parte norcentral de Nicaragua y Validación de trampas artesanales de luz para el control de adultos en Almaciguera y Miraflor, Estelí, Nicaragua. Tesis de Ingeniero Agrónomo., Universidad Nacional Agraria. Managua, Nicaragua. $153 \mathrm{p}$

CATIE (Centro Agronómico Tropical de Investigacion y Enseñanza). 2009. Proyecto Cacao Centroamérica (PCC). Turrialba, CR.

CAMBio - Mercados Centroamericanos para la Biodiversidad. 2015. Manual deOportunidades: Oferta de cacao amigable con la biodiversidad en Centro América. Managua, Nicaragua. 19p.

CBI (Center for the Promotion of Imports from Developing Countries). 2012. Análisis de la cadena de valor de Cacao en Nicaragua. Rotterdan, Holanda. 49 p.

King A.B.S. 1984. Biology and identification of white grubs (Phyllophaga) of economic importance in Central America. Tropical Pest Management, 30(1):36-50.

King A.B.S; Saunders JL. 1984. The invertebrate pests of annual food crops in Central America. A guide to their recognition and control. TDRI, CATIE, Costa Rica, 166 p. 64 lams.

Maes, JM.; Morón, MA. 2017. Melolonthinae (Coleóptera: Melolonthidae) de Nicaragua. Parte 1. Especies reportadas en Nicaragua. Museo Entomológico, Asociación Nicaragüense de Entomología. Revista Nicaragüense de Entomología. León, NI. 291 p.

PROMIPAC. 2006. Niveles y umbrales de daños económicos de las plagas. Segunda Edición; SICA-ZAMORANO. 2006. Tegucigalpa, Honduras. 63p.

RAS (Red de Agrocultura Sostenible). 2011. Lista de Plaguicidas prohibidos. Ciudad de Guatemala, GU. 8 p. 2017. Lista RAS para la gestión de plaguicidas.

Robbins, P; Argüello, H; Zeiss, M. 1997. Effect of three pheromones using amino acids for to capture of May beetles (Phyllopaga spp) in Honduras and Nicaragua. Entomological Society American. November, 1998. Las Vegas Nevada, EE.UU.

UTZ. 2015. Lista de Plaguicidas prohibidos y lista de plaguicidas en vigilancia (v.1.0). UTZ, Departamento de Estándares y Certificación de Ruyterkade. Ámsterdam, Holanda. 19 p. 